\title{
Pulse-coupled resonate-and-fire models
}

$\operatorname{AUTHOR}(\mathrm{S})$ :

Miura, K; Okada, M

\section{CITATION:}

Miura, K ...[et al]. Pulse-coupled resonate-and-fire models. Physical Review E 2004, 70(2): 021914.

ISSUE DATE:

2004-08

URL:

http://hdl.handle.net/2433/49895

RIGHT:

Copyright 2004 American Physical Society 
PHYSICAL REVIEW E 70, 021914 (2004)

\title{
Pulse-coupled resonate-and-fire models
}

\author{
Keiji Miura $^{1,2, *}$ and Masato Okada ${ }^{2,3}$ \\ ${ }^{1}$ Department of Physics, Graduate School of Sciences, Kyoto University, Kyoto 606-8502, Japan \\ ${ }^{2}$ Laboratory for Mathematical Neuroscience, RIKEN Brain Science Institute, Saitama 351-0198, Japan \\ 3 “Intelligent Cooperation and Control," PRESTO, JST, clo RIKEN BSI, Saitama 351-0198, Japan
}

(Received 20 April 2004; published 30 August 2004)

\begin{abstract}
We analyze two pulse-coupled resonate-and-fire neurons. Numerical simulation reveals that an antiphase state is an attractor of this model. We can analytically explain the stability of antiphase states by means of a return map of firing times, which we propose in this paper. The resultant stability condition turns out to be quite simple. The phase diagram based on our theory shows that there are two types of antiphase states. One of these cannot be seen in coupled integrate-and-fire models and is peculiar to resonate-and-fire models. The results of our theory coincide with those of numerical simulations.
\end{abstract}

DOI: 10.1103/PhysRevE.70.021914

PACS number(s): 87.19.La, 87.18.Sn, 05.45.Xt

\section{INTRODUCTION}

The integrate-and-fire model [1] is well known in the context of spiking neuron models. However, it cannot reproduce voltage oscillations near the equilibrium state and resonance in response to sinusoidal current inputs seen in the HodgkinHuxley model [2-4]. Damped oscillations of membrane potential are reported experimentally for biological neurons too [5]. Although we need a model with more than two variables to reproduce these phenomena [6], in general, it is difficult to solve models with more than two variables analytically.

Izhikevich suggested the resonate-and-fire model, which can reproduce voltage oscillations and resonance and is still analytically tractable [2]. The resonate-and-fire model is a two-dimensional linear dynamical system with a threshold. It can be derived as a linearization of the FitzHugh-Nagumo model [7,8] or the Morris-Lecar model [9] about their fixed points. There is another way of simplification of the FitzHugh-Nagumo model or the Morris-Lecar model. The McKean model [10] can be derived as a piecewise linear idealization of the nullclines of the FitzHugh-Nagumo model and Tonnelier and Gerstner derived the piecewise linear Morris-Lecar model in a similar way $[11,12]$. Note that these models are different from the resonate-and-fire model we consider in this paper.

Voltage oscillations can play an important role in transmitting signals in the brain. For example, Izhikevich pointed out that resonance provided an effective tool in selective communication [2-4]. Therefore we need to find out whether a network of resonate-and-fire models has properties that cannot be observed in integrate-and-fire models. The network properties of resonate-and-fire-like models (linearized FitzHugh-Nagumo models) have been investigated [13], but only an oscillatory regime where individual neurons fire spontaneously was considered and subthreshold voltage oscillations were not focused on.

In this paper, we analyze a system of two excitatory or inhibitory pulse-coupled resonate-and-fire models. We found

\footnotetext{
*Electronic address: kmiura@brain.riken.jp
}

that the system settled into an antiphase state in both excitatory and inhibitory coupled cases in numerical simulations. We theoretically evaluated the stability of antiphase states in detail. The system of pulse-coupled integrate-and-fire models has already been investigated [14], and it has been proved that for almost all initial conditions the system evolves to an in-phase state. This contrasts with resonate-and-fire models where the system does not necessarily evolve to an in-phase state.

In Sec. II we briefly review the resonate-and-fire model and its properties. We then suggest a system for two pulsecoupled resonate-and-fire models.

In Sec. III we demonstrate the existence of an antiphase state and construct a theory that can be used to determine the region of existence for antiphase states and assess their stability. We suggest an effective method of calculating the region. We show that global stability of antiphase states can be determined by return maps and their local stability can be determined by a simple equation.

In Sec. IV, using the method proposed in Sec. III, we calculate a phase diagram for the existence and local stability of antiphase states. Stability switches near the boundary of the region of existence or at points where coupling strength is zero. We also determine stability by direct numerical simulations, and the results coincide with those of our theory. The phase diagram indicates there are two types of antiphase states. The antiphase state with a longer period utilizes postinhibitory spiking [2] which cannot be seen in integrateand-fire models.

\section{MODEL}

A resonate-and-fire model is a two-dimensional linear dynamical system with a threshold,

$$
\begin{gathered}
\frac{d x}{d t}=-x-10 y+I, \\
\frac{d y}{d t}=10 x-y,
\end{gathered}
$$

where $x$ and $y$ are internal state variables and $I$ is an external input. We assume that all the variables are dimensionless for 
$\mathrm{I}=1$

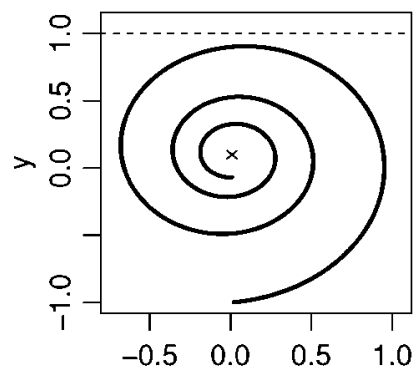

(a)

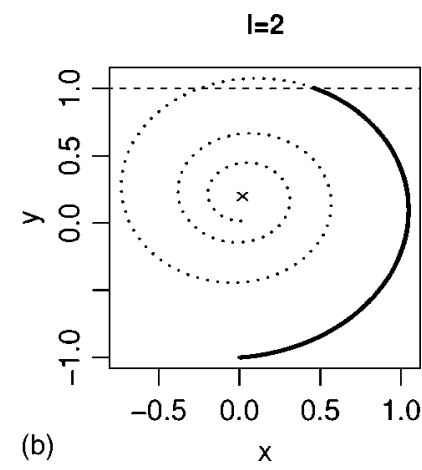

(b)

FIG. 1. Solutions for the resonate-and-fire model. Orbits on the $x y$ plane are plotted. The cross represents a fixed point and the dashed line is threshold. (a) Orbit for $I=1$. (b) Orbit for $I=2$. Neuron is immediately reset to $(x, y)=(0,-1)$ after it exceeds threshold. The dotted line is the orbit a neuron follows if not reset.

simplicity. If $y$ exceeds the threshold $(y=1)$, the internal state is reset to $(0,-1)$. Figure 1 shows typical solutions. The neuron with $I=1$ does not fire, while the neuron with $I=2$ exceeds the threshold and fires. There is a critical value $I_{C}^{1}$ $=1.56$, and neurons with $I>I_{C}^{1}$ can fire.

The resonate-and-fire model has a fixed point satisfying

$$
\begin{gathered}
0=\frac{d x}{d t}=-x-10 y+I, \\
0=\frac{d y}{d t}=10 x-y .
\end{gathered}
$$

The fixed point is $(x, y)=\left(\frac{I}{101}, \frac{10 I}{101}\right)$. When $I>I_{C}^{2}=10.1$, the fixed point is above the threshold and there is no stationary state. The eigenvalues of a linearized system at the fixed point are $-1 \pm 10 i$. Since the real part of the eigenvalue is negative, the fixed point is always stable if it exists. The imaginary part represents the angular velocity around the fixed point.

In this paper, we analyze a system of two pulse-coupled resonate-and-fire models,

$$
\begin{gathered}
\frac{d x_{i}}{d t}=-x_{i}-10 y_{i}+I+K \sum \delta\left(t-t_{k}\right) \quad(i=1,2), \\
\frac{d y_{i}}{d t}=10 x_{i}-y_{i}
\end{gathered}
$$

where $K$ is coupling strength. A neuron receives a pulse in the $x$ direction at the moment another neuron exceeds the threshold $(y=1)$. The neuron that has fired is immediately reset to $(x, y)=(0,-1)$. Our reset value is different from the original value, $(x, y)=(0,1)$, in Izhikevich [2]. The reason is that with the original value, autocatalytic growth in the firing rate would accelerate indefinitely, leading to an explosion of the system. To avoid this, we chose a reset value that did not lie directly on the threshold. Since a neuron with our reset value has a refractory period, our reset value is suitable for a neuron model.
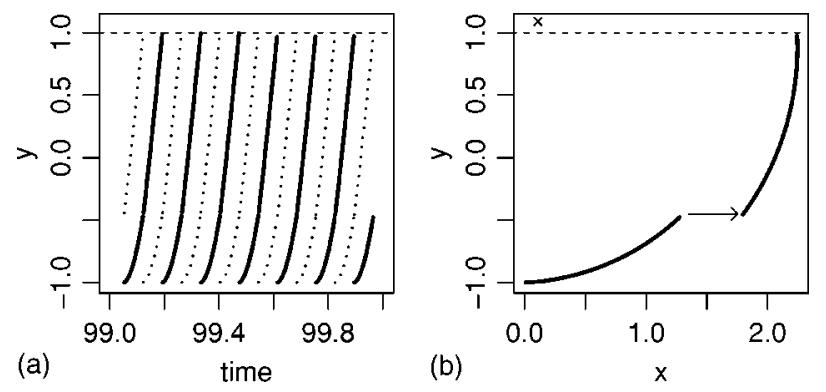

FIG. 2. Example of antiphase states. $K=0.5, I=11$. (a) Time evolution of $y$. Horizontal axis represents time and vertical axis represents y. Solid lines and dotted lines represent each neuron. The dashed line is the threshold $(y=1)$. Two neurons fire alternately at regular intervals. (b) Orbits in $x y$ plane. The arrow indicates a jump in the $x$ direction by a pulse input and the cross denotes a fixed point, $(x, y)=\left(\frac{11}{101}, \frac{110}{101}\right)$. The dashed line is the threshold line. Here, both neurons follow the same orbit, so two orbits overlap.

\section{THEORY OF ANTIPHASE STATES}

\section{A. Antiphase states}

We simulated the system of coupled resonate-and-fire models with $K=0.5, I=11$, and randomized initial conditions and found that it evolves to the state shown in Fig. 2(a). The solid lines and the dotted lines in the figure represent each neuron. The dashed line is the threshold $(y=1)$. The two neurons fire alternately at regular intervals. Since each neuron fires periodically, a phase can be defined with period $2 \pi$. We describe the firing time of one neuron as phase 0 , which evolves in proportion to time. Here, the time at which another neuron fires is described by phase $\pi$. We call this state an antiphase state. In general, we refer to a state where two neurons follow the same orbit but have phase shift $\pi$ as an antiphase state [15-17].

Figure 2(b) plots the orbits in the $x-y$ plane for the same data as in Fig. 2(a). The arrow indicates a jump in the $x$ direction by a pulse input and the cross denotes a fixed point, $(x, y)=(I / 101,10 I / 101)$. The dashed line is the threshold. Here, both neurons follow the same orbit, so the two orbits overlap in the figure. In the following, we theoretically examine in what parameter regions antiphase states exist stably.

\section{B. Existence of antiphase states}

Before we discuss the stability of antiphase states, we derived a theory about the region of existence of antiphase states.

We can obtain the solution orbits of antiphase states analytically. Since the resonate-and-fire model is linear except for the moment of firing, we can integrate it piecewise. For ease of explanation, consider the imaginary plane and define

$$
z \equiv x+i y \text {. }
$$

Then, the resonate-and-fire model can be written as

$$
\frac{d}{d t}\left(z-z_{*}\right)=(-1+10 i) z+I=(-1+10 i)\left(z-z_{*}\right),
$$

where 


$$
z_{*}=\frac{I}{101}+\frac{10 I}{101} i .
$$

We can integrate it easily to

$$
z(t)=z_{*}+\left(z_{0}-z_{*}\right) e^{(-1+10 i) t},
$$

where $z_{0}$ denotes an initial condition at $t=0$. For simplicity, let us consider an orbit that is reset at $t=0$. The reset value is $(x, y)=(0,-1)$. Therefore the initial condition is $z_{0}=-i$ in the complex plane.

We assume the orbit receives a pulse input and jumps in the $x$ direction at $t=T$. Let $t=T$ in Eq. (7) and add $K$. Then, the neuronal state just after the pulse input becomes

$$
z(T+0)=z_{*}+\left(-i-z_{*}\right) e^{(-1+10 i) T}+K .
$$

Then, the orbit evolves to $t=T+T^{\prime}$. The orbit at $t=T+T^{\prime}$ is written using Eq. (8) as

$$
z\left(T+T^{\prime}\right)=z_{*}+\left[z(T+0)-z_{*}\right] e^{(-1+10 i) T^{\prime}} .
$$

The threshold line is $y=1$ and the imaginary part of $z$ is $y$. Thus the condition for firing time is

$$
y\left(T+T^{\prime}\right)=\operatorname{Im}\left[z\left(T+T^{\prime}\right)\right]=1 .
$$

The orbit with $T^{\prime}=T$ matches a situation where two neurons fire alternately at regular intervals. Thus the condition that the orbit is an antiphase state is

$$
y(2 T)=1 .
$$

Next, to set a limit to the $T$ range satisfying $y(2 T)=1$ theoretically, we used the following theorem.

Theorem 1. Given the following assumptions: (1) the neurons are in an antiphase state (as defined in Sec. III A) and (2) one of the neurons from the pair has received a pulse input, then the following must be true. The neuron receiving the input must fire within a $360^{\circ}$ rotation around the fixed point.

Note that the antiphase state is obtained from the firing times of the two neurons while the angle of the rotation around the fixed point may be defined without reference to the firing times. The proof is as follows. The radius of an orbit shrinks with time. If a neuron does not fire within $360^{\circ}$ rotation, it cannot fire forever. Therefore a neuron in an antiphase state must fire within a $360^{\circ}$ rotation after a pulse input.

This theorem restricts the $T$ range in which to search to a finite region. The restricted region is $0<T<\frac{2 \pi}{10}$ because the angular velocity around the fixed point is always 10 and it takes $\frac{2 \pi}{10}$ to rotate by $360^{\circ}$.

Thus we should find a $T$ value satisfying $y(2 T)=1$ in $0<T<\frac{2 \pi}{10}$, where the orbit must reach the threshold at time $2 T$ for the first time after being reset. The condition that the orbit exceeds the threshold at time $2 T$ for the first time after being reset can be mathematically represented as $y(t)<1$ in $0<t<2 T$.

\section{Stability of antiphase states}

In this section we derive a theory for the stability of antiphase states.
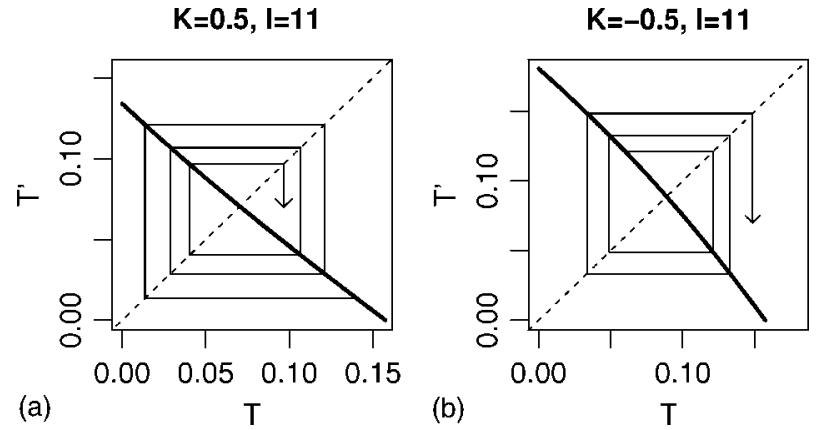

FIG. 3. (a) The bold line represents the return map with $K$ $=0.5$ and $I=11$. The dashed line is diagonal. The thin line with an arrow is construction to obtain firing times. The antiphase state is stable. (b) Return map with $K=-0.5$ and $I=11$. The antiphase state is unstable.

$T^{\prime}$ satisfying $y\left(T+T^{\prime}\right)=1$ [Eq. (10)] can be obtained as a function of $T \cdot y\left(T+T^{\prime}\right)=1$ can be explicitly written as

$$
\begin{aligned}
\frac{10 I}{101}+ & K e^{-T^{\prime}} \sin \left(10 T^{\prime}\right)-e^{-T^{\prime}-T} \cos \left[10\left(T^{\prime}+T\right)\right] \\
& -\frac{I e^{-T^{\prime}-T}}{101}\left\{10 \cos \left[10\left(T^{\prime}+T\right)\right]+\sin \left[10\left(T^{\prime}+T\right)\right]\right\}=1 .
\end{aligned}
$$

Here $T^{\prime}$ denotes the interval between pulse input and firing and $T$ denotes the interval between reset and pulse input. We refer to the map that maps $T$ to $T^{\prime}$ as a return map. Figure 3 (a) plots the return map with $K=0.5$ and $I=11$.

In antiphase states, the interval between pulse input and firing for one neuron equals the interval between reset and pulse input for another neuron. Thus we can obtain the firing times of both neurons by using the return map iteratively. The thin line with the arrow in Fig. 3(a) demonstrates how to iterate the return map.

The intersection between the return map and diagonal line represents an antiphase state and is a fixed point on the return map. For the return map in Fig. 3(a), the orbit finally arrives at the fixed point starting from any initial value and the antiphase state is globally stable. However, Fig. 3(b) demonstrates that the antiphase state with $K=-0.5$ and $I=11$ is unstable.

Although we can determine global stability by drawing return maps, we have to redraw them when we change the values of $K$ and $I$. Therefore it is difficult to monitor global stability over the whole range of $K$ and $I$. In the following, we limit our focus to local stability and show that the condition of neutral stability is analytically obtained over the whole range of $K$ and $I$. Local linear stability is determined as described below. We calculate the slope of the return map (bold line) at the fixed point. An antiphase state is stable if the absolute value of the slope is smaller than 1. Otherwise, it is unstable. When the slope is -1 , an antiphase state destabilizes through the period doubling bifurcation [18-21]. When the slope is 1, a pair of antiphase states disappears through saddle-node bifurcation. 
We already derived the theory to determine the region of existence of antiphase states in the previous section. Here, we will derive a condition for the neutral stability of antiphase states.

We define $f\left(T, T^{\prime}\right)$ as a function of $T$ and $T^{\prime}$,

$$
f\left(T, T^{\prime}\right) \equiv y\left(T+T^{\prime}\right)=\operatorname{Im}\left[z\left(T+T^{\prime}\right)\right]
$$

where $y\left(T+T^{\prime}\right)$ is defined in Eq. (10). An antiphase state is neutrally stable if the slope of its return map at the fixed point is -1 , i.e.,

$$
\frac{d T^{\prime}}{d T}=-\frac{\left(\frac{d f}{d T}\right)}{\left(\frac{d f}{d T^{\prime}}\right)}=-1,
$$

where the derivative is evaluated at the fixed point. After additional calculations, the condition for neutral stability [Eq. (14)] becomes

$$
K\left[\tan \left(10 T^{\prime}\right)-10\right]=0 .
$$

Note that Eq. (15) only depends on the sign of $K$ and $T^{\prime}(=T)$, and can be rewritten as

$$
\begin{gathered}
K=0, \\
T=\arctan (10) / 10=0.1471128, \quad \text { and } \\
\mathrm{T}=[\arctan (10)+\pi] / 10=0.461272,
\end{gathered}
$$

where the principal value is chosen. An antiphase state is neutrally stable if any of the above three conditions are satisfied. Although arctan is a multivalued function, the theorem in the previous section restricts the $T$ range to $0<T<\frac{2 \pi}{10}$ and we only need to consider two values of $T$.

We can rewrite the three conditions in terms of $K$ and $I$ by setting $T=T^{\prime}=0.1471128$ (or 0.461272 ) in Eq. (10). As a result, the three conditions for neutral stability become

$$
\begin{gathered}
K=0, \\
I=-5.056553 K+1.587449, \text { and } \\
I=4.58563 \mathrm{~K}+4.461462 .
\end{gathered}
$$

\section{RESULTS}

We calculated the phase diagram for the existence and stability of antiphase states in the KI plane based on our theory. The results are plotted in Fig. 4. The antiphase state is stable in the region marked "S" and unstable in that marked "U." In the region marked "S\&U" there are two types of antiphase states. One of these is stable and the other is not. The dashed line represents neutral stability [Eq. (19)]. There are no antiphase states in the dark region. In the dark region, we found two types of solutions. One is the resting state where both neurons stay at the fixed point. The other is the state where one of the neurons repetitively fires while the other neuron oscillates under the threshold.

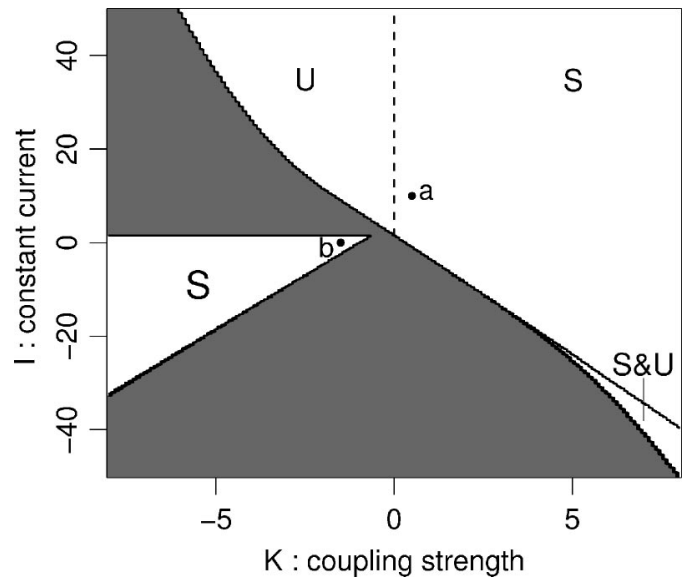

FIG. 4. Existence and stability of antiphase states. In regions marked "S" or "U," the antiphase state is stable or unstable. In the region marked "S\&U," there are two types of antiphase states. One of these is stable and the other is not. The dashed line is neutral stability $(K=0)$. There is no antiphase state in the dark region.

To verify the theoretically obtained phase diagram in Fig. 4, we did a numerical simulation using the Runge-Kutta method for numerous parameter values. To reduce the computational error, we used a modified Runge-Kutta method $[22,23]$ at the moment an orbit crosses the threshold, where we approximate the orbit by a line and obtain a firing time with the accuracy of $O\left(d t^{2}\right)$. Thus the order of the computational error at the threshold is $O\left(d t^{2}\right)$. We adopted the second-order Runge-Kutta method because the fourth-order Runge-Kutta method does not improve the order of the computational error in total. The computational error with the second-order Runge-Kutta method is $O\left(d t^{3}\right)$ in each step and the number of steps is $O(1 / d t)$. Therefore the total computational error except at the threshold is $O\left(d t^{2}\right)$. This is exactly the same order as the computational error at the threshold [23]. Therefore we cannot improve the computational error if we use the fourth-order Runge-Kutta method. We did numerical simulations on 17500 lattice points in the KI plane. We chose $K$ from -9.9 to 9.9 with a fixed step size of 0.2 and $I$ from -70 to 70 with a fixed step size of 0.8 . We assessed an antiphase state as stable if the orbit starting from it remained as it was. The results of the numerical simulations coincided with those of our theory for all the parameter values.

There are two distinct stable regions in Fig. 4. To determine the properties of these regions, we checked the orbits of antiphase states at points marked "a" and "b" in Fig. 4. The results are in Figs. 5(a) and 5(b).

Figure 5(a) plots the orbit of an antiphase state with $K$ $=0.5$ and $I=10$. The main characteristics of the orbit are that $K>0$ and period $T$ is relatively short. Since a pulse input just accelerates firing time, the orbit is not unique to coupled resonate-and-fire models and can also be seen in coupled integrate-and-fire models. The arrow indicates a jump in the $x$-direction by a pulse input and the cross denotes the fixed point. The dashed line is the threshold $(y=1)$. The period of this type of antiphase state cannot be longer than 0.1471128 because the antiphase state becomes unstable at $T$ $=0.1471128$ as shown in Eq. (17). 

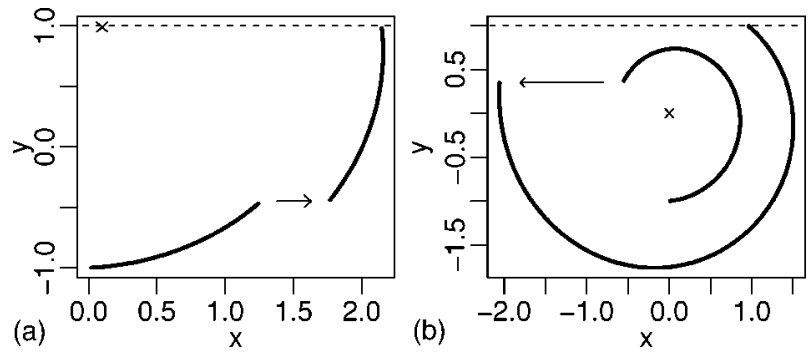

FIG. 5. Orbits of antiphase states at points marked "a" and "b" in Fig. 4. The arrow indicates a jump in the $x$ direction by a pulse input and the cross denotes a fixed point. The dashed line is threshold $(y=1)$. (a) Orbit with $K=0.5$ and $I=10$. (b) Orbit with $K=-1.5$ and $I=0$.

Figure 5(b) plots the orbit of the antiphase state with $K$ $=-1.5$ and $I=0$. The main characteristics of the orbit are that $K<0$ and period $T$ is relatively long. The orbit jumps in the negative direction by a pulse input and rebounds to fire as if it were a spring. This is unique to coupled resonate-and-fire models and cannot be seen in coupled integrate-and-fire models. In the region with $I>I_{c}^{1}=1.56$, there is no antiphase state such as this because a neuron spontaneously fires without pulse inputs there. The period of this type of antiphase state cannot be shorter than $\frac{\pi}{10}$. In the limit that $K$ approaches $-\infty, T$ approaches $\frac{\pi}{10}$. However, it can be shown that there is no antiphase state with $T=\frac{\pi}{10}$ if $K$ is finite. Therefore $T=\frac{\pi}{10}$ gives a lower limit.

Figure 6 is the magnification of Fig. 4 around $(K, I)$ $=(0,1.56)$. The figure illustrates that stability changes near the boundary of the region of existence and on line $K=0$. In the region marked "S," the antiphase state is stable and unstable in "U." The two dashed lines represent neutral stability [Eqs. (19) and (20)]. There is no antiphase state in the dark region.

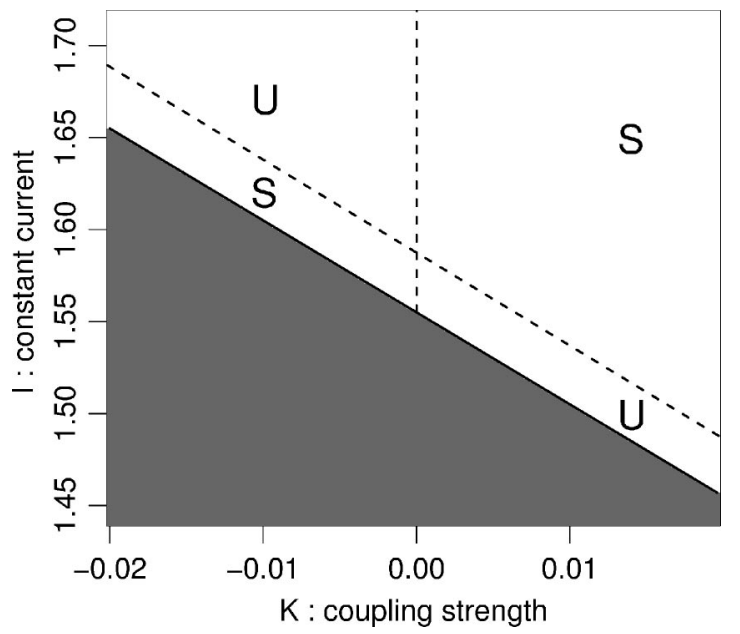

FIG. 6. Magnification of Fig. 4 around $(K, I)=(0,1.56)$. In the regions marked "S" or "U," the antiphase state is theoretically stable or unstable. Two dashed lines represent neutral stability [Eqs. (19) and (20)]. There is no antiphase state in the dark region. Stability changes near the boundary of the region of the existence and on line $K=0$.

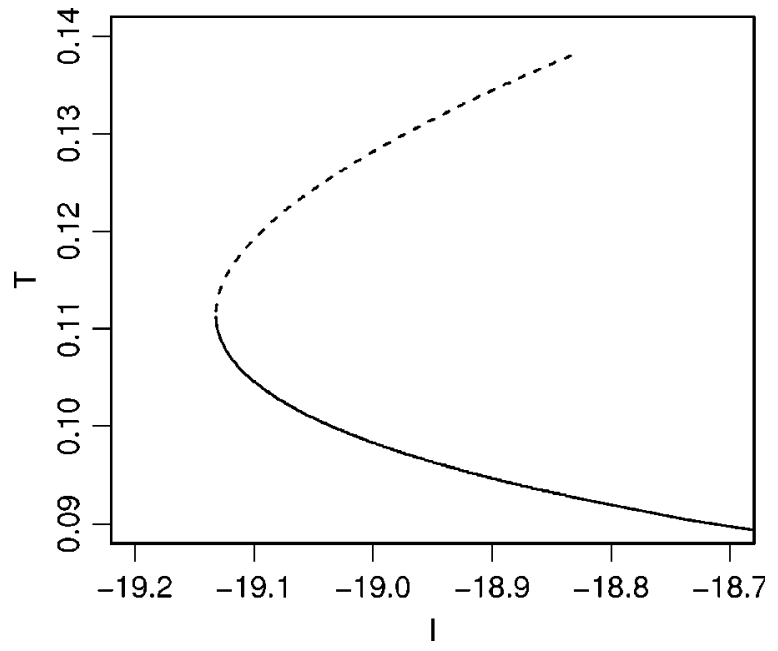

FIG. 7. Period of antiphase states at $K=4$ as a function of $I$. Two states coexist in the region. The solid line and dashed line denote stable state and unstable state, respectively.

Two antiphase states coexist in the region marked "S\&U" in Fig. 4. To examine this region, we computed the period of antiphase states at $K=4$ as a function of $I$. The results are plotted in Fig. 7. The solid line denotes a stable state and the dashed line denotes an unstable one. At $I=-19.13$, two antiphase states are created pairwise through the saddle-node bifurcation [18-21]. In $-19.13<I<-18.83$, the two states coexist. At $I=-18.83$, the unstable state disappears because the orbit of the unstable state becomes tangential to the threshold. In $I>-18.83$, the orbit exceeds the threshold before the orbits of unstable antiphase state are formed; that is, if an orbit is not reset at the first time that the orbit exceeds the threshold and reset at the second time, then the orbit becomes an antiphase state. However, this state is imaginary and there is no unstable state. In $I>-18.83$, only the stable state exists.

Figure 8 plots the period of antiphase states as a function of $I$ at different values of $K$. The thick solid line denotes a stable state and the thick dashed line denotes an unstable one. The line with $K=4$ is the same as that in Fig. 7. Although the period changes continuously with $K$, we plotted only four lines for brevity. Instead, we plotted the envelopes of the bifurcation points for all the $K$ values. There are three types of bifurcation and the three thin lines represent the envelopes. Bifurcation points are obtained by $m=-1, \infty$, and 1. Here $m$ is defined as the left-hand side of Eq. (14), i.e., $m=\frac{d T^{\prime}}{d T}$. Note that $m$ depends on $K$. At $m=1$, two antiphase states are created pairwise through the saddle-node bifurcation of the return map. At $m=-1$, the antiphase state destabilizes through the period doubling bifurcation. Here, the condition that $m=-1$ becomes Eq. (17). At $m=\infty$, the antiphase state disappears because the orbit is at a tangent to the threshold. In $K>K_{c}(=1.31)$, two antiphase states with different periods can coexist for some $I$ ranges. One of these is stable and the other is not. In $K<K_{c}$, there is only one antiphase state and it is stable if $T<0.1471128$. This is consistent with Fig. 6 . Note that the condition $m=-1$ can be written as $T=0.1471128$.

In $K<0$ and $I<I_{c}^{1}(=1.56)$, there is also a region where two antiphase states coexist and there is an unstable region 


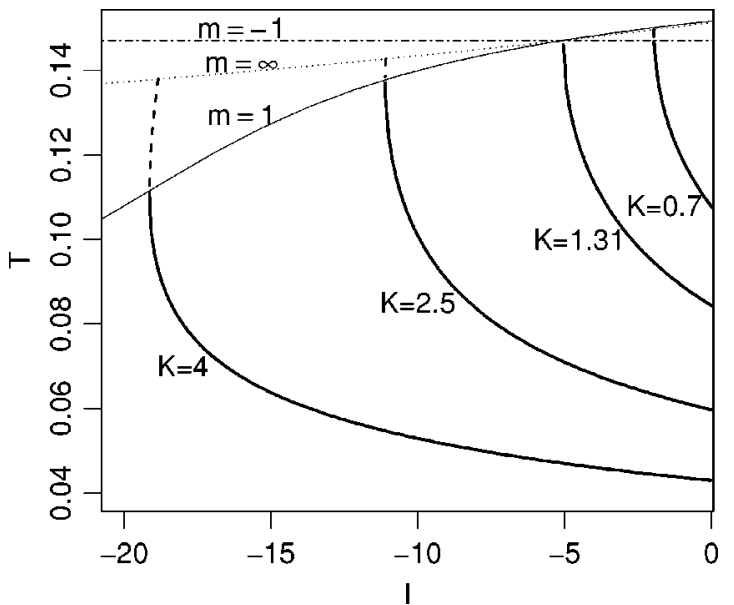

FIG. 8. Thick lines denote periods of antiphase states as functions of $I$ at different values of $K(K=0.7,1.31,2.5,4)$. The solid line and dashed line denote the stable state and unstable state, respectively. Three thin lines are $m=-1, \infty$, and 1 , where $m$ is defined as the left-hand side of Eq. (14), i.e., $m=d T^{\prime} / d T$. $m$ depends on $K$ and these lines are envelopes of the bifurcation points.

near the boundary of the region of existence, as in $K>0$. However, these regions are so small that we cannot see them in Fig. 4.

\section{SUMMARY AND DISCUSSION}

In this paper, we analyzed a system for two pulse-coupled resonate-and-fire models. We found that the system settled to an antiphase state in numerical simulations. We looked for the existence and evaluated the stability of antiphase states. We found an effective method of calculating the region of existence, where we set limits for the region theoretically and then utilized analytically obtained orbits. We found that stability of antiphase states could be determined by means of a return map of firing times. The condition for neutral stability turned out to be unexpectedly simple [Eq. (15)]. Based on our theory, we calculated a phase diagram for the existence and local stability of antiphase states.

Stability changed near the boundary of the region of existence or at $K=0$. We also determined stability by direct numerical simulations, and the results coincided with that of our theory. The phase diagram revealed that there were two types of antiphase states. An antiphase state with a longer period is unique to coupled resonate-and-fire models and cannot be seen in coupled integrate-and-fire models.

We modeled a spike as a delta function. The case where a spike is modeled as an alpha function has been studied in [13]. However, the stability condition was not obtained explicitly, and a phase diagram for existence of antiphase states was not calculated as a function of coupling strength. Therefore it is difficult to compare their results with ours.

In this paper, we focused on antiphase states. What about in-phase states? If two neurons start under the same initial conditions, they must continue to follow the same orbit. Then, in-phase states exist at $I>I_{c}^{1}(=1.56)$ where a neuron can fire spontaneously. However, in-phase states are unstable against perturbations. This can be explained as follows. Let the orbits of two neurons differ infinitesimally. When the neurons fire in succession, the leading neuron receives a pulse input from the following neuron just after being reset, while the following neuron receives a pulse input from the leading neuron just before firing. The positions where they receive pulse inputs differ considerably. The effects of pulse inputs on orbits are so different that the difference between orbits becomes finite. Thus in-phase states are always unstable.

\section{ACKNOWLEDGMENTS}

We are grateful to S. Shinomoto and Y. Kuramoto for valuable discussions and suggestions about this work. This work was partially supported by Grant-in-Aid for Scientific Research on Priority Areas No. 14084212.
[1] C. Koch, Biophysics of Computation (Oxford University Press, Oxford, 1999).

[2] E. M. Izhikevich, Neural Networks 14, 883 (2001).

[3] E. M. Izhikevich, N. S. Desai, E. C. Walcott, and F. C. Hoppensteadt, Trends Neurosci. 26, 161 (2003).

[4] E. M. Izhikevich, BioSystems 67, 95 (2002).

[5] R. R. Llinas, Science 242, 1654 (1988).

[6] E. M. Izhikevich, Int. J. Bifurcation Chaos Appl. Sci. Eng. 10, 1171 (2000).

[7] A. F. Fitzhugh, Biophys. J. 1, 445 (1961).

[8] J. Nagumo, S. Arimoto, and S. Yoshizawa, Proc. IRE 50, 2061 (1962)

[9] L. Morris and H. Lecar, Biophys. J. 35, 193 (1981).

[10] M. P. McKean, Adv. Math. 4, 209 (1970).

[11] A. Tonnelier and W. Gerstner, Phys. Rev. E 67, 021908
(2003).

[12] A. Tonnelier, SIAM (Soc. Ind. Appl. Math.) J. Appl. Math. 63, 459 (2003).

[13] A. D. Garbo, M. Barbi, and S. Chillei, Int. J. Bifurcation Chaos Appl. Sci. Eng. 11, 2549 (2001).

[14] R. E. Mirollo and S. H. Strogatz, SIAM (Soc. Ind. Appl. Math.) J. Appl. Math. 50, 1645 (1990).

[15] A. Pikovsky, M. Rosenblum, and J. Kurths, Synchronization (Cambridge University Press, Cambridge, England, 2001).

[16] Y. Kuramoto, Chemical Oscillations, Waves and turbulence (Springer, Berlin, 1984).

[17] A. T. Winfree, The Geometry of Biological Time (Springer, Berlin, 2001).

[18] J. Guckenheimer and P. Holmes, Nonlinear Oscillations, Dynamical Systems and Bifurcations of Vector Fields (Springer, 
Berlin, 1983).

[19] I. A. Kuznetsov and Y. A. Kuznetsov, Elements of Applied Bifurcations Theory (Springer, Berlin, 1998).

[20] F. C. Hoppensteadt and E. M. Izhikevich, Weakly Connected Neural Networks (Springer, Berlin, 1997).
[21] S. Wiggins, Introduction to Applied Nonlinear Dynamical Systems and Chaos (Springer, Berlin, 1990).

[22] D. Hansel, G. Mato, C. Meunier, and L. Neltner, Neural Comput. 10, 467 (1998).

[23] M. J. Shelley and L. Tao, J. Comput. Neurosci. 11, 111 (2001). 